\title{
Diagnosing cardiac amyloidosis: A wealth of new possibilities with nuclear cardiac imaging
}

\author{
Renée P. Bullock-Palmer, MD FACC FASNC FASE FSCCT ${ }^{\mathrm{a}}$ \\ a Department of Cardiology, Deborah Heart and Lung Center, Browns Mills, NJ
}

Received Apr 18, 2019; accepted Apr 19, 2019

doi:10.1007/s12350-019-01740-w

\section{See related article, pp. 209-218}

\section{PATHOPHYSIOLOGY OF CARDIAC AMYLOIDOSIS}

Amyloidoses are a group of protein-folding disorders in which more than one organ is infiltrated by proteinaceous deposits known as amyloid. The deposits are derived from one of several amyloidogenic precursor proteins, and the prognosis of the disease is determined both by the organ(s) involved and the type of amyloid. Amyloid involvement of the heart, cardiac amyloidosis (CA), carries the worst prognosis of any involved organ, and light-chain amyloidosis (AL-CA) is the most serious form of the disease. ${ }^{1} \mathrm{CA}$ may be due to myocardial deposition of transthyretin protein derived from the liver known as transthyretin cardiac amyloidosis (TTR-CA) or may be due to AL-CA with myocardial deposition of immunoglobulin light-chain proteins derived from a clone of plasma cells. ${ }^{2}$

The average age of onset of AL-CA is 50 years of age. ${ }^{1}$ The prevalence of AL-CA is similar in males and females and multiorgan involvement usually occurs. ${ }^{1}$ TTR-CA has significant cardiac involvement when compared with AL-CA and is usually associated with bilateral carpal tunnel syndrome and neuropathy. ${ }^{1}$ TTRCA may be wild type (normal) transthyretin (TTRCAwt) or mutant (hereditary) transthyretin (TTR-CAm). TTR-CAwt has a marked male predominance with a male to female prevalence ratio of $15: 1$ and usually occurs at $65+$ years of age. ${ }^{1}$ TTR-CAwt has marked

Reprint requests: Renée P. Bullock-Palmer, MD FACC FASNC FASE FSCCT, Department of Cardiology, Deborah Heart and Lung Center, Browns Mills, NJ; renee.palmer@gmail.com

J Nucl Cardiol 2021;28:219-24.

1071-3581/\$34.00

Copyright (C) 2019 American Society of Nuclear Cardiology. cardiac involvement and is usually preceded by carpal tunnel syndrome by 5 to 10 years. ${ }^{1}$ TTR-CAm usually occurs at a younger age of $40+$ years and may occur in either males or females with a slight male predominance. ${ }^{1}$ There is a common African American variant of TTR-CAm which has a prevalence of 3 to $4 \%$ in the United States African American population and is due to a mutation in the V122 1 gene. ${ }^{1}$ This variant of TTRCAm usually occurs at an older age, 60 to 65 years, when compared to the other forms of TTR-CAm. ${ }^{1}$

\section{CLINICAL FEATURES OF CARDIAC AMYLOIDOSIS}

Clinical features suggestive of CA are recurrent unexplained heart failure particularly in African Americans males $>60$ years old with preserved left ventricular ejection fraction $(\mathrm{HFpEF})$, an unexplained increase in left ventricular wall thickness $(>12 \mathrm{~mm})$ and a restrictive left ventricular filling pattern suggestive of increased filling pressures. There is usually left atrial dysfunction in the absence of atrial fibrillation and an abnormal global longitudinal strain (GLS) with " apical sparing," pattern. ${ }^{1}$ On cardiac magnetic resonance imaging (Cardiac MRI), late gadolinium enhancement usually occurs with CA. ${ }^{1}$ Of note echocardiography and Cardiac MRI are unable to differentiate TTR-CA from AL-CA.

\section{DIAGNOSIS OF TTR-CA WITH NUCLEAR CARDIAC IMAGING: THE DAWN OF A NEW ERA FOR PATIENTS WITH TTR-CA}

Over the last few years there has been an increasing interest in diagnosing TTR-CA non-invasively with Nuclear Cardiac imaging. This interest was further heightened in 2016 with the release of the American Society of Nuclear Cardiology Practice Points document on the use of technetium-99m pyrophosphate $(99 \mathrm{~m}$ TcPYP) in diagnosing TTR-CA. ${ }^{3}$ 
Over 3 decades ago it was shown that the bone avid radiotracer $99 \mathrm{~m}$ Tc-PYP can be used to non-invasively diagnose CA. ${ }^{4,5}$ In 2005 it was shown by Perugini, et al. that another bone avid radiotracer technetium- $99 \mathrm{~m}$ 3,3diphosphono-1,2-propanodicarboxylic acid (99m TcDPD) scintigraphy could be used to differentiate TTRCA from AL-CA with a sensitivity and specificity of $100 \%$ as $99 \mathrm{~m}$ Tc-DPD had an affinity for the amyloid fibrils in TTR-CA while this was not the same for patients with AL-CA. ${ }^{6}$ The Perugini scale of the degree of radiotracer uptake of $99 \mathrm{~m}$ Tc-DPD into the myocardium assessed by single photon emission computed tomography (SPECT) (Table 1) was also derived from this study. ${ }^{6}$ However, $99 \mathrm{~m}$ Tc-DPD is not approved by the Food and Drug Administration for use in the United States (US). In 2013 Bokhari et al. had shown that in the US the widely available radiotracer, $99 \mathrm{~m}$ Tc-PYP could be used with scintigraphy to non-invasively differentiate TTR-CA from AL-CA with a sensitivity of $97 \%$ and a specificity of $100 \%$ utilizing a quantitative heart to contralateral lung $(\mathrm{H} / \mathrm{CL})$ ratio of greater than 1.5 assessed on planar imaging. ${ }^{7}$ Moreover, Gillmore et al. had shown that when $99 \mathrm{~m}$ Tc-PYP scintigraphy was performed along with serum and urine clonal analysis to exclude monoclonal gammopathy, the combined findings of grade 2 or 3 myocardial radiotracer uptake on scintigraphy and the absence of a monoclonal protein in serum or urine had a specificity and positive predictive value for TTR-CA of $100 \%$ (positive predictive value confidence interval, 98.0 to 100$){ }^{8}$ This study had proposed that TTR-CA could be reliably diagnosed with this method without the need for endocardial biopsy. ${ }^{8}$

These studies have proven the utility of $99 \mathrm{~m} \mathrm{Tc}$ PYP/DPD in non-invasively diagnosing TTR-CA with great accuracy (Figure 1). This has changed the diagnostic paradigm for this disease that was once thought to be difficult to diagnose resulting in many patients being undiagnosed and therefore inappropriately treated. Therefore, many of these patients had very poor outcomes. However, this novel application of old technology in nuclear cardiac imaging to reliably diagnose TTR-CA has ignited great research interest in this area (Table 2).

\section{SENSITIVITY OF 18F-SODIUM FLUORIDE (NAF) POSITRON EMISSION TOMOGRAPHY (PET) FOR THE IMAGING OF CA}

In this edition of the Journal, Martineau et al. examined the sensitivity of $18 \mathrm{~F}$-sodium fluoride $(\mathrm{NaF})$ positron emission tomography (PET) for the imaging of CA. ${ }^{9}$ In this pilot study a cohort of 15 patients (7 patients with TTR-CA, 4 patients with AL-CA and 4 patient controls) were retrospectively reviewed. Qualitative interpretation and quantitative analyses with average left ventricular standardized uptake value (SUVmean) and target-to-background-ratios (TBRmean) were performed. TBRmean was significantly increased in patients with TTR-CA while SUVmean was not. SUVmean was also poor at differentiating TTR-CA from AL-CA or controls. Additionally, quantitative assessment using a TBRmean cut off value of 0.89 had a sensitivity and specificity for diagnosing TTR-CA of $75 \%$ and $100 \%$, respectively. However, qualitative interpretation yielded a sensitivity and specificity for diagnosing TTR-CA of $57 \%$ and $100 \%$, respectively. The study investigators had concluded that while quantitative analysis using $18 \mathrm{~F}-\mathrm{NaF}$ PET/CT demonstrated good diagnostic accuracy, the low TBRmean observed in TTR-CA indicates a poor myocardial signal. This had led to their conclusion that $18 \mathrm{~F}-\mathrm{NaF}$ PET/CT is not yet ready for clinical use in diagnosing TTR-CA until there are further comparative studies with 99m Tc-PYP/99m Tc-DPD.

This study had a few limitations which included the fact that the study was a retrospective single center study with a small cohort of patients. Therefore, these study findings should not be extrapolated to a larger patient population until further research is done with a larger

Table 1. Perugini score and visual description of myocardial radiotracer uptake of technetium $99 \mathrm{~m}-$ 3,3-diphosphono-1,2-propanodicarboxylic acid (99m Tc-DPD) on single photon emission computed tomography (SPECT) ${ }^{6}$

\section{Perugini score}

\section{Visual description}

Grade 0

Grade 1

Grade 2

Grade 3
No myocardial uptake and normal bone uptake

Myocardial uptake less than rib uptake

Myocardial uptake equal to rib uptake

Myocardial uptake greater than rib uptake with mild/absent rib uptake 


\section{American Society of Nuclear Cardiology (ASNC) Practice 2016 Points document on use of $99 \mathrm{mTc}$-PYP to diagnose TTR-CA*}

Gillmore, et al had shown that the combined findings of $99 \mathrm{mTc}$-PYP radiotracer myocardial uptake and the absence of a monoclonal protein in serum or urine had a specificity and positive predictive value (PPV) for TTR-CA of $100 \%(\mathrm{Cl}-98.0-100)$

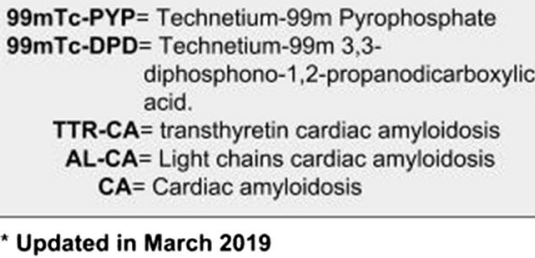

* Updated in March 2019

Bokhari, et al had shown that $99 \mathrm{mTc}$-PYP could be used with scintigraphy to non-invasively differentiate TTR-CA from AL-CA with a sensitivity of $97 \%$ and a specificity of $100 \%$ a sensitivity and specificity of $100 \%$

\begin{tabular}{|c|c|c|}
\hline \multirow[t]{2}{*}{1987} & \multicolumn{2}{|c|}{$\begin{array}{l}\text { Gertz, et al showed that } \\
99 \mathrm{mTC}-\mathrm{PYP} \text { could be used to } \\
\text { non-invasively diagnose CA }\end{array}$} \\
\hline & 1982 & $\begin{array}{l}\text { Wizenberg, et al showed } \\
\text { that } 99 \mathrm{mT} \text { T-PYP could } \\
\text { be used to non-invasively } \\
\text { diagnose CA }\end{array}$ \\
\hline
\end{tabular}

Figure 1. Development of scientific evidence establishing technetium 99m PYP/DPD as a reliable method for diagnosing TTR-CA non-invasively. An outline of the development of scientific evidence over the past 37 years showing the diagnostic utility of bone avid radiotracers such as technetium-99m Pyrophosphate (99m Tc-PYP) and technetium-99m 3,3-diphosphono-1,2-propanodicarboxylic acid (99m Tc-DPD) in diagnosing transthyretin cardiac amyloidosis (TTR-CA).

Table 2. Comparison of the sensitivity and specificity of technetium-99m pyrophosphate $(99 \mathrm{~m}$ TcPYP), technetium-99m-3,3-diphosphono-1,2-propanodicarboxylic acid (99m Tc-DPD), and 18Fsodium fluoride $(\mathrm{NaF})$ in diagnosing transthyretin cardiac amyloidosis (TTR-CA)

\begin{tabular}{llcccc} 
Study & Radiotracer & Number of patients & Sensitivity (\%) & Specificity (\%) & p value \\
\hline Bokhari et al. $^{7}$ & 99m Tc-PYP & 45 & 97 & 100 & $<0.0001$ \\
Perugini et al. $^{6}$ & 99m Tc-DPD & 25 & 100 & 100 & $<0.05$ \\
Martineau et al. $^{9}$ & 18F-NaF & 11 & 75 & 100 & 0.026 \\
\hline
\end{tabular}

group of patients. The TBRmean for TTR-CA being less than 1 indicates a poor myocardial signal for $18 \mathrm{~F}-\mathrm{NaF}$ which results in a lower sensitivity in detecting TTR-CA of $75 \%$ by quantitative analysis and $57 \%$ by qualitative interpretation. Therefore, there is limited clinical utility for $18 \mathrm{~F}-\mathrm{NaF}$ when compared to $99 \mathrm{~m} \mathrm{Tc}-\mathrm{PYP} / \mathrm{DPD}$ both of which have a higher sensitivity for diagnosing TTRCA of $>95 \%$. ${ }^{6,7}$ The study investigators acknowledged the lower sensitivity of $18 \mathrm{~F}-\mathrm{NaF}$ PET/CT to diagnose TTR-CA particularly with qualitative assessment (57\%) vs the quantitative assessment (75\%). This difference in the sensitivity in the quantitative assessment compared with the qualitative interpretation was used to justify the systematic acquisition of rest perfusion imaging in all subjects undergoing $18 \mathrm{~F}-\mathrm{NaF}$ PET/CT study for assessment of $\mathrm{CA}$ to provide more accurate myocardial contours which were used for quantitative analyses. The lower sensitivity of $18 \mathrm{~F}-\mathrm{NaF}$ in detecting TTR-CA compared with $99 \mathrm{~m}$ Tc-PYP/DPD may also be due to the inability of $18 \mathrm{~F}-\mathrm{NaF}$ PET/CT to diagnose TTR-CA early in the disease stage. ${ }^{10}$ Additionally, the other potential causes for this variable myocardial uptake of $18 \mathrm{~F}-\mathrm{NaF}$ radiotracer may also be due to the etiology of CA (wild type vs mutant) and to the composition of the calcium matrix. ${ }^{10}$ The study investigators were not able to identify features associated with potential false negative studies due to the small study cohort that did not allow for rigorous statistical comparison of these variables.

Based on these study findings, 18F-NaF PET/CT has a much lower sensitivity in detecting TTR-CA compared with $99 \mathrm{~m}$ Tc-PYP/DPD (75\% vs > 95\%), Table $1^{6,7,9}$ and therefore cannot be recommended for 


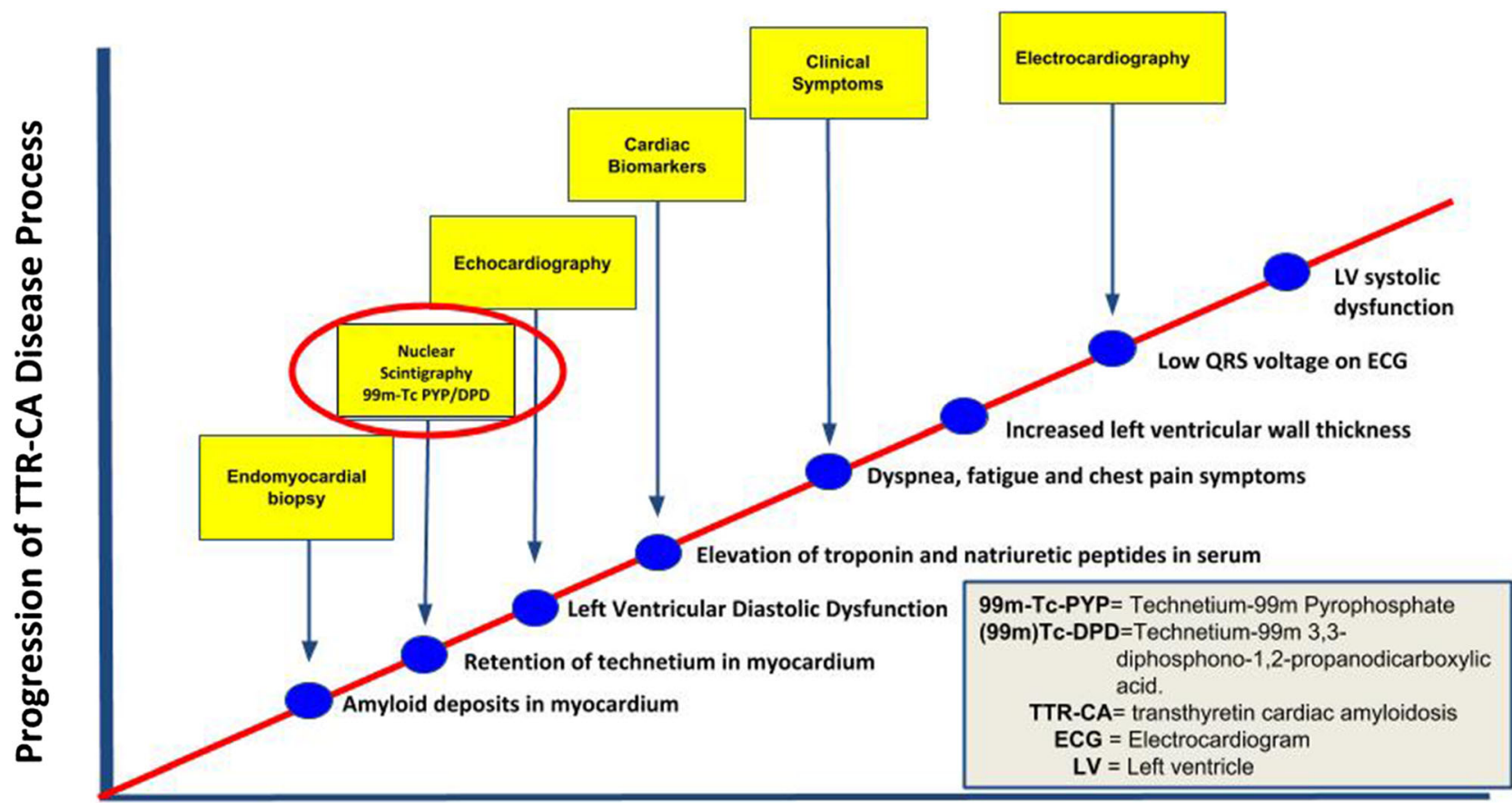

Time from onset of transthyretin (TTR) amyloid myocardial deposits (years)

Figure 2. TTR-CA cascade - an outline of transthyretin cardiac amyloidosis (TTR-CA) cascade showing the progression of the TTR-CA disease process over time and the diagnostic tools that can be useful during this disease progression. It is important to note that Nuclear Scintigraphy with technetium-99m Pyrophosphate (99m Tc-PYP) or technetium-99m 3,3-diphosphono-1,2-propanodicarboxylic acid (99m Tc-DPD) detects the disease process very early even before echocardiography and elevation of cardiac laboratory biomarkers.

routine clinical use in diagnosing TTR-CA until larger scale studies are performed.

\section{TTR-CA CASCADE AND PROGNOSIS}

During the early onset of TTR-CA there is early deposition of amyloid deposits in the myocardium and this gradually increases over time. The diagnostic strength of bone avid radiotracers such as $99 \mathrm{~m}$ TcPYP/DPD is their ability to detect TTR-CA early in the disease process. ${ }^{6,7,11}$ As the disease progresses with increased amyloid deposition the left ventricular myocardium becomes thicker and stiffer resulting in a restrictive left ventricular myocardial filling pattern. ${ }^{1}$ This can be detected on echocardiography with spectral and tissue Doppler imaging along with global longitudinal strain assessment with the typical apical sparing pattern. This apical sparing pattern has a sensitivity of $93 \%$ but a lower specificity of $82 \%{ }^{12}$ when compared to $99 \mathrm{~m}$ Tc-PYP/DPD with a specificity of $100 \% .^{6-8}$ In the early stage of the disease functional class remains normal as patients are usually asymptomatic, however as the disease progresses with increased myocardial deposition, New York Heart Association (NYHA) functional class begins to decline and there is an increase in serum biomarkers such as troponin and brain natriuretic peptide (BNP) levels. ${ }^{11}$ As NYHA functional class progresses to class II/III the left ventricular ejection fraction shortly thereafter begins to decline. ${ }^{11}$ Additionally, changes on electrocardiography with decreased QRS voltage pattern may be apparent. ${ }^{1}$ However, it is important to note that the low-voltage QRS complexes are far less common in TTR-CA than in AL-CA, despite a greater amyloid burden in the heart. ${ }^{1}$ The timeline of these diagnostic and clinical characteristics are summarized in Figure 2 outlining the "TTR-CA Cascade".

The development of the novel use of bone avid radiotracers 99m Tc-PYP and 99m Tc-DPD has changed the diagnostic paradigm of TTR-CA which was once thought to be challenging to diagnose and sometimes the diagnosis was made much later in the disease process or was oftentimes undiagnosed. The utilization of these bone avid radiotracers to diagnose TTR-CA have led to the ability to detect this disease early in the process. This early diagnosis of TTR-CA could potentially facilitate early treatment of the disease and improve the overall 
prognosis for these patients who were once thought to not have many therapeutic options. In fact the median age of survival from time of diagnosis based on the TRACS study was approximately 25 months in TTRCAm and 43 months in TTR-CAwt. ${ }^{11}$ Additionally, the usual causes of death include sudden death, heart failure, and sepsis. ${ }^{11}$ Therefore, early diagnosis and treatment of this disease is vital in the improvement of the prognosis for patients with TTR-CA.

\section{NOVEL THERAPEUTIC OPTIONS ON THE HORIZON FOR TTR-CA}

There are three potential targets for treatment of patients with TTR-CA. These three potential targets include suppression of TTR synthesis, TTR stabilization, and TTR fibril degradation and absorption. ${ }^{11}$ TTR synthesis can be suppressed by liver transplantation. ${ }^{11}$ However there are 2 potential therapeutic medications that have been studied and have been shown to decrease TTR synthesis. ${ }^{11}$ These 2 agents that have been shown to decrease TTR synthesis are Patisiran ${ }^{13}$ and Inotersen. ${ }^{14}$ Patisiran was shown in the APOLLO clinical trial to improve multiple clinical manifestations of hereditary transthyretin amyloidosis (TTR-CAm). ${ }^{13}$ Inotersen was shown in the NEURO-TTR study to improve the course of neurologic disease and quality of life in patients with TTR-CA. ${ }^{14}$ Side effects of thrombocytopenia and glomerulonephritis should be managed with enhanced monitoring. ${ }^{14}$ Tafamidis was shown in the ATTR-ACT study to be a TTR stabilizer that decreased all-cause mortality, cardiovascular related hospitalization rate, and the decline in functional capacity in patients with TTR-CA. ${ }^{15}$ Fibril degradation and reabsorption in patients with TTR-CA can be achieved with several therapeutic agents such as doxycycline, Tauroursodeoxycholic Acid (TUDCA), and anti-serum amyloid protein (SAP) antibody. ${ }^{11}$

With the development of these treatment options in the pipeline, this has given hope for improved outcomes in patients with TTR-CA.

\section{FUTURE DIRECTIONS FOR TTR-CA MANAGEMENT}

\section{Monitoring of Treatment}

With emerging therapeutic options for patients with TTR-CA there is a need for a reliable method for detecting disease progression and monitoring improvement of disease state with treatment. Currently, there is a paucity of research data on the use of serial imaging with bone avid radiotracers in the detection of disease progression or improvement of disease state with treatment. ${ }^{2}$ A small single center study involving 20 patients and use of $99 \mathrm{~m}$ Tc-PYP did not show any significant change in myocardial radiotracer retention despite clinical progression of the disease as determined by NYHA functional class, lab biomarkers, echocardiographic parameters, progression to cardiac transplantation, and/or death. ${ }^{2}$ Quantitative assessment with echocardiography and global longitudinal strain, CMR with tissue characterisation using native T1 mapping and extracellular volume fraction (ECV) as well as amyloid imaging with PET tracers may play an important role in monitoring of disease progression as well as disease improvement on therapy. ${ }^{2}$ However, more research is necessary in this area to determine the best diagnostic strategy to monitor disease progression or regression.

\section{Need for Amyloidosis Centers of Excellence}

The development and establishment of a reliable diagnostic paradigm for patients with TTR-CA with the use of bone avid radiotracers such as $99 \mathrm{~m}$ Tc-PYP and $99 \mathrm{~m}$ Tc-DPD has led to the development of new treatment options in the pipeline for these patients. The next phase is the development of multidisciplinary Amyloidosis Centers of Excellence nationwide as the prevalence of TTR-CA is likely to increase tremendously over the next few years. It is vital that the diagnosis is made accurately and timely and that these patients are followed closely and treated appropriately. The multidisciplinary team should include an experienced cardiac imaging specialist, an experienced heart failure specialist with access to advanced heart failure therapies such as left ventricular assist devices (LVAD) and experience in heart failure transplantation, an experienced interventional cardiologist is also necessary particularly when endomyocardial biopsy may be required in selected patients. A cardiothoracic surgeon with experience in LVAD implantation and heart transplantation should also be included on the team. There should also be a hematologist/oncologist who is experienced in diagnosing and treating AL-CA included in this multidisciplinary team. A hepatologist with experience in liver transplantation should also be included in selected cases of TTR-CA where liver transplantation may have to be considered.

\section{NUCLEAR IMAGING AND TRANSFORMATION OF THE DIAGNOSTIC AND TREATMENT PARADIGM FOR TTR-CA}

The establishment of the use of bone avid radiotracers such as $99 \mathrm{~m}$ Tc-PYP/DPD to accurately diagnose TTR-CA with a sensitivity and specificity greater than 
95\% have transformed the diagnostic paradigm for this disease that was once considered a terminal diagnosis that often times remained undiagnosed. This transformation of the diagnostic paradigm has led to a wealth of therapeutic options in the pipeline for these patients and have given these patients a new ray of hope.

\section{Disclosure}

Renée P. Bullock-Palmer has nothing to disclose.

\section{References}

1. Falk RH, Alexander KM, Liao R, Dorbala S. AL (light-chain) cardiac amyloidosis: A review of diagnosis and therapy. J Am Coll Cardiol 2016;68(12):1323-41.

2. Singh V, Falk R, Di Carli MF, Kijewski M, Rapezzi C, Dorbala S. State-of-the-art radionuclide imaging in cardiac transthyretin amyloidosis. J Nucl Cardiol 2019;26(1):158-73.

3. Dorbala S, Bokhari S, Miller E, Bullock-Palmer RP, Soman P, Thompson R. American Society of Nuclear Cardiology (ASNC) practice points on technetium- $99 \mathrm{~m}$ pyrophosphate imaging for transthyretin cardiac amyloidosis-1st edition in 2016, updated in 2019.

4. Wizenberg TA, Muz J, Sohn YH, Samlowski W, Weissler AM. Value of positive myocardial technetium-99m-pyrophosphate scintigraphy in the noninvasive diagnosis of cardiac amyloidosis. Am Heart J 1982;103(4 Pt 1):468-73.

5. Gertz MA, Brown ML, Hauser MF, Kyle RA. Utility of technetium Tc $99 \mathrm{~m}$ pyrophosphate bone scanning in cardiac amyloidosis. Arch Intern Med 1987;147(6):1039-44.

6. Perugini E, Guidalotti PL, Salvi F, Cooke RM, Pettinato C, Riva $\mathrm{L}$, et al. Noninvasive etiologic diagnosis of cardiac amyloidosis using 99mTc-3,3-diphosphono-1,2-propanodicarboxylic acid scintigraphy. J Am Coll Cardiol 2005;46(6):1076-84.
7. Bokhari S, Castaño A, Pozniakoff T, Deslisle S, Latif F, Maurer MS. (99m)Tc-pyrophosphate scintigraphy for differentiating lightchain cardiac amyloidosis from the transthyretin-related familial and senile cardiac amyloidoses. Circ Cardiovasc Imaging 2013;6(2):195-201.

8. Gillmore JD, Maurer MS, Falk RH, Merlini G, Damy T, Dispenzieri A, et al. Nonbiopsy diagnosis of cardiac transthyretin amyloidosis. Circulation 2016;133(24):2404-12.

9. Martineau P, Finnerty V, Giraldeau G, Authier S, Harel F, Pelletier-Galarneau M. Examining the sensitivity of $18 \mathrm{~F}-\mathrm{NaF}$ PET for the imaging of cardiac amyloidosis. J Nucl Cardiol 2019. https://d oi.org/10.1007/s12350-019-01675-2.

10. Ng QKT, Sethi P, Saunders TA, Pampaloni MH, Flavell RR. Discordant findings on $18 \mathrm{~F}-\mathrm{NaF}$ and $99 \mathrm{mTc}-\mathrm{HDP}$ Bone scans in a patient with ATTR cardiac amyloidosis. Clin Nucl Med 2018;43(3):e89-92.

11. Castaño A, Drachman BM, Judge D, Maurer MS. Natural history and therapy of TTR-cardiac amyloidosis: Emerging diseasemodifying therapies from organ transplantation to stabilizer and silencer drugs. Heart Fail Rev 2015;20(2):163-78.

12. Phelan D, Collier P, Thavendiranathan P, Popović ZB, Hanna M, Plana JC, et al. Relative apical sparing of longitudinal strain using two-dimensional speckle-tracking echocardiography is both sensitive and specific for the diagnosis of cardiac amyloidosis. Heart 2012;98(19):1442-8.

13. Adams D, Gonzalez-Duarte A, O'Riordan WD, Yang CC, Ueda $\mathrm{M}$, Kristen AV, et al. Patisiran, an RNAi therapeutic, for hereditary transthyretin amyloidosis. N Engl J Med 2018;379(1):11-21.

14. Benson MD, Waddington-Cruz M, Berk JL, Polydefkis M, Dyck $\mathrm{PJ}$, Wang AK, et al. Inotersen treatment for patients with hereditary transthyretin amyloidosis. N Engl J Med 2018;379(1):22-31.

15. Maurer MS, Schwartz JH, Gundapaneni B, Elliott PM, Merlini G, Waddington-Cruz $\mathrm{M}$, et al. Tafamidis treatment for patients with transthyretin amyloid cardiomyopathy. $\mathrm{N}$ Engl J Med 2018;379(11):1007-16.

Publisher's Note Springer Nature remains neutral with regard to jurisdictional claims in published maps and institutional affiliations. 